\title{
Shaping the Infant Microbiome With Non-digestible Carbohydrates
}

\author{
Stella Verkhnyatskaya ${ }^{1}$, Michela Ferrari ${ }^{1}$, Paul de Vos $^{2}$ and Marthe T. C. Walvoort ${ }^{1 *}$ \\ ${ }^{1}$ Stratingh Institute for Chemistry, Faculty of Science and Engineering, University of Groningen, Groningen, Netherlands, \\ ${ }^{2}$ University Medical Center Groningen, Groningen, Netherlands
}

OPEN ACCESS

Edited by:

F. Javier Moreno,

Instituto de Investigación en Ciencias de la Alimentación (CIAL), Spain

Reviewed by:

Francisco J. Plou, Instituto de Catálisis y

Petroleoquímica (ICP), Spain

Susana Delgado, Instituto de Productos Lácteos de Asturias (IPLA-CSIC), Spain

*Correspondence: Marthe T. C. Walvoort m.t.c.walvoort@rug.n

Specialty section:

This article was submitted to Systems Microbiology,

a section of the journal

Frontiers in Microbiology

Received: 23 November 2018 Accepted: 08 February 2019

Published: 25 February 2019

Citation:

Verkhnyatskaya S, Ferrari M, de Vos P and Walvoort MTC (2019) Shaping the Infant Microbiome With

Non-digestible Carbohydrates.

Front. Microbiol. 10:343

doi: 10.3389/fmicb.2019.00343
Natural polysaccharides with health benefits are characterized by a large structural diversity and differ in building blocks, linkages, and lengths. They contribute to human health by functioning as anti-adhesives preventing pathogen adhesion, stimulate immune maturation and gut barrier function, and serve as fermentable substrates for gut bacteria. Examples of such beneficial carbohydrates include the human milk oligosaccharides (HMOs). Also, specific non-digestible carbohydrates (NDCs), such as galacto-oligosaccharides (GOS) and fructo-oligosaccharides (FOS) are being produced with this purpose in mind, and are currently added to infant formula to stimulate the healthy development of the newborn. They mimic some functions of $\mathrm{HMO}$, but not all. Therefore, many research efforts focus on identification and production of novel types of NDCs. In this review, we give an overview of the few NDCs currently available [GOS, FOS, polydextrose (PDX)], and outline the potential of alternative oligosaccharides, such as pectins, (arabino)xylo-oligosaccharides, and microbial exopolysaccharides (EPS). Moreover, state-of-the-art techniques to generate novel types of dietary glycans, including sialylated GOS (Sia-GOS) and galactosylated chitin, are presented as a way to obtain novel prebiotic NDCs that help shaping the infant microbiome.

Keywords: infant, microbiome, non-digestible carbohydrates, exopolysaccharides, transglycosylation

\section{INTRODUCTION}

Humans live in symbiosis with trillions of bacteria, and most of them are symbionts and beneficial to the host (Sender et al., 2016). Disturbance in our microbiota can contribute to the development of many diseases (Wang et al., 2017). Bacteria are mainly present in the areas that are more exposed to the surrounding environment such as the skin, vaginal and oral mucosa, and the GIT. The gut microbiota has been extensively studied due to its impact on the establishment of immunity (Martin et al., 2010) and prevention of chronic inflammation (Belkaid and Hand, 2014). While the fetal GIT was considered sterile for many years, emerging evidence suggests that colonization of the GIT starts already at the prenatal stage with neonatal colonization by Enterobacter, Escherichia, Shigella, and Staphylococcus species, as detected in the umbilical cord, placenta, and amniotic fluid (Carmen Collado et al., 2016). After birth, the newborn gut is rapidly colonized by different bacterial

Abbreviations: APS, acidic polysaccharides; AXOS, (arabino-)xylo-oligosaccharides; DP, degree of polymerization; EPS, exopolysaccharides; FOS, fructo-oligosaccharides; GHs, glycosyl hydrolases; GIT, gastrointestinal tract; GMP, glycomacropeptide; GOS, galacto-oligosaccharides; GTs, glycosyl transferases; H-APS, high-molecular weight acidic polysaccharides; HePS, heteropolysaccharides; HMOs, human milk oligosaccharides; HoPS, homopolysaccharides; NDCs, non-digestible carbohydrates; NEC, necrotizing enterocolitis; NPS, neutral polysaccharides; PDX, polydextrose; POS, pectin oligosaccharides; SB-POS, sugar beet pulp pectin oligosaccharides; SCFAs, short chain fatty acids. 
strains with the first colonizers being facultative aerobes such as Escherichia and Enterococcus, whose oxygen consumption allows colonization of anaerobic bacteria, with the most abundant being Bifidobacterium (Houghteling and Walker, 2015). Many early-life factors have an impact on the composition of the infant gut microbiota, including the mode of delivery, the infant feeding pattern, diet composition, and the use of antibiotics, but also the health of the mother during pregnancy (Gonzalez-Perez et al., 2016).

The early colonization process is crucial for a healthy microbiome and prevents disease later in life. Gut microbiota are essential for digestion of food, but also to function as a barrier against pathogens, and for the development of immune tolerance to innocuous antigens and microorganisms (Yang et al., 2016). Imbalances in the intestinal microbiome composition can result in bacterial overgrowth or lower species diversity, making the host more susceptible to pathogenic infections (Lozupone et al., 2012). Furthermore, microbial dysbiosis may lead to autoimmune and allergic diseases. The healthy infant intestinal microbiome has a low microbial diversity, with Bifidobacterium, Bacteroidetes, Firmicutes, and Proteobacteria being most abundant. Feeding has a major influence on the microbiota composition, as breast-fed infants have higher Bifidobacterium and Enterobacteria numbers and a lower diversity in comparison to formula-fed infants (Milani et al., 2017).

There is a growing understanding of the mechanisms by which a balanced microbiome contributes to health. For instance, many genera such as Eubacterium and Bacteroides are involved in the production of vitamin K (Rossi et al., 2011), an essential cofactor promoting the $\gamma$-carboxylation of glutamate residues involved in blood clotting (Gröber et al., 2014). Bifidobacterium species are able to produce folate, a vitamin involved in DNA synthesis and repair with an undisputed importance in neurological development (Crider et al., 2012), with the best producing strains being Bifidobacterium adolescentis and Bifidobacterium pseudocatenulatum (Rossi et al., 2011). Lactobacilli carry the rib operon, which is implicated in the de novo synthesis of riboflavin, which is important in developmental processes and in the hemopoietic system (Thakur et al., 2016). Moreover, gut microbiota are responsible for the production of SCFAs, such as acetate, propionate, and butyrate. Acetate is the most abundant, and it is used by many gut commensals to produce propionate and butyrate in a growth-promoting cross-feeding process. SCFAs are important for the reduction of the intestinal $\mathrm{pH}$ and the consequent inhibition of pathogen's adhesion. Moreover, butyrate is the preferred energy source for colon epithelial cells, where it contributes to the maintenance of the gut intestinal barrier, exerts immunomodulatory and anti-inflammatory effects (Stilling et al., 2016; Zhang et al., 2018), also through epigenetic mechanisms (Furusawa et al., 2013; Paparo et al., 2014), and may even prevent colorectal cancer (Wu et al., 2018).

A healthy infant microbiome is normally created under the guidance of molecules in human milk. This is mainly accomplished by HMOs, which serve as feed for specific bacterial species. HMOs are a family of $>200$ structurally different molecules that vary in quantity and composition from mother to mother, and over the course of lactation. However, some general trends in HMO composition are present (Table 1). HMOs are composed of a linear or branched backbone containing galactose (Gal), $N$-acetylglucosamine (GlcNAc), and glucose (Glc), which can be decorated with fucose (Fuc) and sialic acid (Sia) residues, and this decoration pattern depends on the mother's secretory status (Bode, 2012). Only members of Bifidobacterium and Bacteroides were shown to metabolize HMOs (Marcobal et al., 2010). Especially Bifidobacterium bifidum and Bifidobacterium infantis are efficient utilizers of HMOs, whereas they are moderately digested by Bifidobacterium breve and Bifidobacterium longum. Interestingly, Bifidobacterium animalis and $B$. adolescentis are incapable of degrading HMOs (LoCascio et al., 2009; Sela and Mills, 2010). To ensure a high number in the gut, bifidobacteria have been observed to create a cross-feeding niche, as the extracellular fermentation of HMOs by $B$. bifidum is associated with a cooperative effect for $B$. infantis, which is able to import the released sugars and digest them intracellularly (Garrido et al., 2012; Thomson et al., 2018).

For infants where human milk is not an option, infant formula supplemented with NDCs that should mimic prebiotic functions of HMOs have been created (Vandenplas et al., 2015). A prebiotic is defined as "a substrate that is selectively utilized by host microorganisms conferring a health benefit" (Gibson et al., 2017). HMOs fulfill these criteria, as they are not digested in the upper part of the GIT of infants (Engfer et al., 2000), while they serve as preferred food source for beneficial bacteria. Next to HMOs, other NDCs or dietary fibers have been shown to be major drivers of gut microbiome composition and function, and might be added to infant formula for this purpose (Benitez-Paez et al., 2016). Interestingly, the currently applied molecules do not mimic all the functions of the $>200$ HMOs found in human milk, so novel oligosaccharides are needed to fill this void. This review aims to inspire the selection of future NDCs that can be added to infant formula by reviewing beneficial glycans that show great promise as modulators of the microbiome, with a focus on their interaction with bifidobacteria and lactobacilli, since most is known about these genera. Moreover, state-of-the-art techniques to generate novel types of dietary glycans are presented.

\section{NDCS CURRENTLY ADDED TO INFANT FORMULA}

To mimic the beneficial effects of HMOs, two alternative oligosaccharides are routinely added to infant formula: GOS and FOS (Table 1). GOS are produced by enzymatic transglycosylation from lactose (vide infra), providing a mixture of differently linked oligosaccharides with a DP from 2 to 8 . The Gal units are linked through $\beta$-galactosidic linkages, which are resistant to GIT enzymes until they reach the colon where they are fermented by bacteria. In general, GOS stimulate the growth of bifidobacteria (Absmanner et al., 2010), and especially the numbers of $B$. adolescentis are impacted (Sierra et al., 2015). FOS are generally produced by enzymatic digestion from naturally isolated inulin, yielding oligosaccharides with DP from 2 to 9, and bifidobacteria readily grow when FOS are used as a sole carbon source (Macfarlane et al., 2008). 
TABLE 1 | Overview of oligosaccharide structures discussed herein.

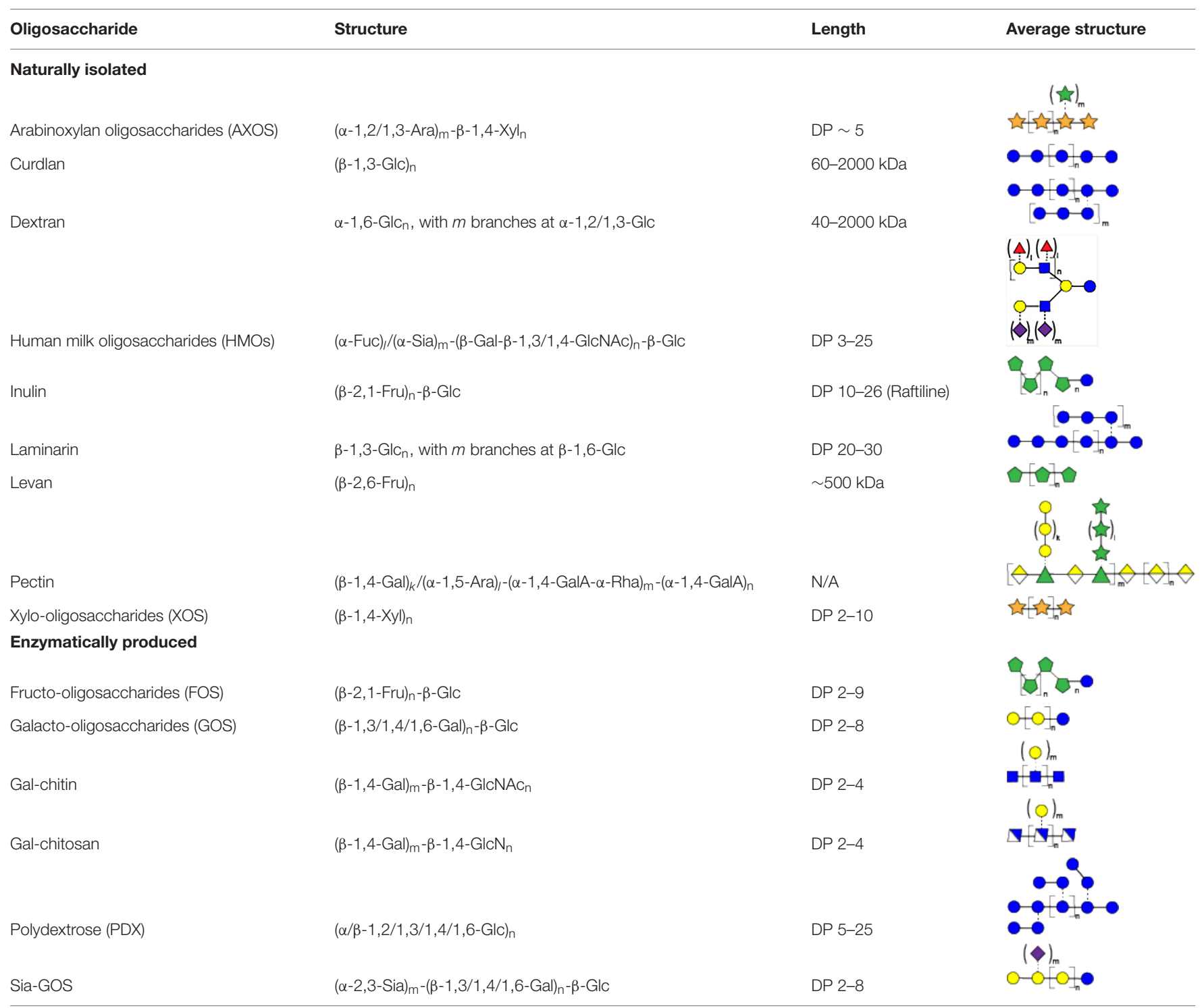

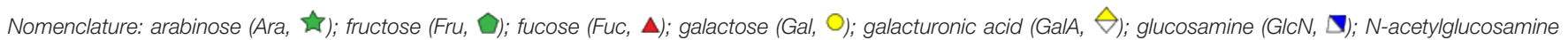

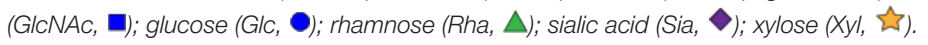

When mixtures of GOS/FOS in a $9 / 1$ ratio are used, the ratio of different Bifidobacterium species was similar to breast-fed infants (Haarman and Knol, 2005). This GOS/FOS mixture was also demonstrated to be the best growth substrate for Bifidobacteria and Lactobacilli, while inulin and PDX led to poor growth (Vernazza et al., 2006). PDX is a synthetic polymer of randomly connected Glc units with an average DP of 12 and all possible glucosidic linkages: $\alpha$ - or $\beta$ - and $1 \rightarrow 2,1 \rightarrow 3$, $1 \rightarrow 4$, and predominantly $1 \rightarrow 6$ (Ramiro do Carmo et al., 2016). When PDX was used in combination with GOS in a 1:1 ratio, the increase in Bifidobacterium species, specifically $B$. infantis, B. longum, and B. catenulatum, was similar to the breast-fed microbiota, where $B$. infantis, $B$. longum, and $B$. breve are predominant (Scalabrin et al., 2012). Interestingly, this GOS/PDX mixture was also identified in a commercial brand of infant formula (Nijman et al., 2018). Next to prebiotic properties, GOS, FOS, and mixtures of both components were also shown to have immunomodulatory properties, which have recently been reviewed (Macfarlane et al., 2008; Ackerman et al., 2017, Akkerman et al., 2018).

\section{ALTERNATIVE NDCS ISOLATED FROM NATURAL SOURCES}

Polysaccharides with prebiotic potential have mostly been extracted from the cell wall of higher plants including cereals and grains, fruits, and vegetables, seaweeds, and microalgae (de Jesus Raposo et al., 2016). In this section, we focus on the naturally isolated polysaccharides POS and AXOS that have 
already been investigated for their prebiotic effect and might serve as alternative for HMOs.

Pectins have received widespread attention for their potential as prebiotics. They are composed of a backbone of galacturonic acids, which are hypothesized to mimic the Sia residues in HMOs (Table 1). Pectins are heteropolysaccharides and are available from citrus peels, apple pomace, sugar beet pulp, and potato pulp. The hydrolysis of pectins yields POS, which are composed of galacturonic acid, galactose, rhamnose, arabinose, and xylose building blocks. Moreover, POS can be methylated or esterified on the galacturonic acid residues, and the degree of methylation, esterification, and the ratios of monosaccharides depends on the source of pectin and the type of extraction method used. In light of this structural diversity, studies with POS become more reliable and reproducible when the exact molecular structure is described. POS has a demonstrated prebiotic effect, promoting the growth of Bifidobacteria and Lactobacilli. Interestingly, especially neutral POS, such as galactan, GOS, arabinan, and arabino-oligosaccharides, enhance the growth of Bifidobacteria to a similar extent as inulin (Onumpai et al., 2011; Di et al., 2017). A similar increase in bifidobacteria numbers was observed for an arabinoserich mixture of SB-POS, while lactobacilli were selectively enhanced using lemon peel waste-derived POS, which was high in galacturonic acids, and the number of bacterial members of Faecalibacterium prausnitzii group and Roseburia intestinalis (both of the phylum Firmicutes) increased with all types of pectins (Gomez et al., 2016). In contrast, a commercial source of SB-POS, which was shown to contain a high galacturonic acid content, had little effect on numbers of bifidobacteria, highlighting the importance of the pectin composition (Leijdekkers et al., 2014). Infant formula with pectins has been studied in human infant trials, but there was no effect of the acidic oligosaccharides on bifidobacteria and lactobacilli (Fanaro et al., 2005).

Xylo-oligosaccharides (XOS, Table 1) are present in fruits, vegetables, bamboo, honey, and milk, and can be produced on an industrial scale by enzymatic degradation of xylanrich materials (Aachary and Prapulla, 2011). XOS is readily fermented by commensal bacteria, and can in humans increase the population of fecal bifidobacteria and SCFA production (Lecerf et al., 2012). AXOS (Table 1) are prepared by degradation of arabinoxylan, which is the major non-cellulose polysaccharide in cereals and plants. In a fermentation study, it was shown that B. longum B24 could liberate the arabinose units from AXOS without degrading the xylan backbone, while B. longum B18 was able to metabolize XOS up to DP4 (Riviere et al., 2018). B. adolescentis B72 degraded various types of FOS, partially degraded inulin, and metabolized XOS longer than DP4. The authors suggested that the strain-specific mechanisms to utilize different glycans lead to a cooperative effect and simultaneous striving of different bacterial strains. A similar cross-feeding effect was observed between B. longum NCC2705 and Eubacterium rectale ATCC 33656 when grown on AXOS (Riviere et al., 2015). B. longum is able to release arabinose and produce acetate, whereas $E$. rectale uses acetate to produce butyrate. When co-cultured on AXOS, the consumption of arabinose by $B$. longum and concomitant release of acetate allowed E. rectale to produce butyrate, resulting in a simultaneous prebiotic and butyrogenic effect (Riviere et al., 2016). Other examples of such a commensal cross-feeding relationship with bifidobacteria have been reported, including Faecalibacterium (De Vuyst and Leroy, 2011; Moens et al., 2016). Negatively charged XOS structures, containing glucuronic acid units, have also been isolated from hardwood (Rivas et al., 2017), and may be promising candidates for novel charged prebiotic NDCs (vide infra).

\section{POTENTIAL OF EXOPOLYSACCHARIDES AS NOVEL NDCS}

Exopolysaccharides produced by Gram-positive bacteria currently attract a great deal of attention because of their wide range of beneficial properties (Ryan et al., 2015). Regularly new EPS structures are identified that have a specific health effect, and especially the immune-modulating properties are often investigated (Castro-Bravo et al., 2018). From recent reviews on the characterized EPS structures of Lactobacillus and Bifidobacterium, their great structural diversity is immediately apparent (Hidalgo-Cantabrana et al., 2014; Castro-Bravo et al., 2018; Oleksy and Klewicka, 2018). They are broadly divided into HoPS, which are composed of a single sugar building block, and HePS, which display a repeating fragment of two to eight different sugar units.

Most HoPS are found to be susceptible to fermentation by commensal bacteria (Salazar et al., 2016), which is presumably directly linked to their relatively simple molecular structure, albeit that they can be very large in size. For instance, the prebiotic effect of $\beta$-fructans was investigated with two levan-type EPS isolated from Lactobacillus sanfranciscensis, and compared with levan (fructan with $\beta-2,6$ linkages, Table 1), inulin (fructan with $\beta-2,1$ linkages), and FOS (Dal Bello et al., 2001). An enrichment of Bifidobacterium species in human fecal samples in a large bowel model medium was observed with the EPS and inulin as added carbon source, while levan and FOS had no effect. This may reflect the importance of both the length of the carbohydrate, and the fructose linkage type in the isolated EPS, which may be different from commercial levan. The capability of Bifidobacterium species to directly metabolize the $L$. sanfranciscensis EPS was further demonstrated in a fermentation study (Korakli et al., 2002). $\beta$-Glucans, including curdlan (linear $\beta-1,3-G l c$, Table 1) and laminarin ( $\beta-1,3 / 1,6-G l c$, Table 1), are also readily fermented by bifidobacteria. Especially the $B$. infantis population benefitted from $\beta$-glucan digestion, and concomitant increased production of propionate and butyrate was observed (Zhao and Cheung, 2011).

In contrast, there is a lack of data on the digestibility of HePS by commensal bacteria, presumably due to their complex structures and generally low isolated yields. Both bifidobacteria and lactobacilli display structurally diverse HePS, which may contain galacto-pyranose and -furanose, rhamnose, mannose, and 6-deoxy-talose, among others 
(Hidalgo-Cantabrana et al., 2014). In a fecal slurry fermentation experiment, the uncharacterized EPS from different $B$. animalis, $B$. pseudocatenulatum, and B. longum species isolated from humans were investigated for their prebiotic effect (Salazar et al., 2008). Although there were high inter-individual variations, the data indicated an EPS-related enrichment of Bifidobacterium species, similar to the result obtained with inulin. Bacteroides fragilis DSMZ 2151 was also found to digest (uncharacterized) HePS from B. longum E44 and B. animalis subsp. lactis $\mathrm{R} 1$, with concomitant increase in propionate and acetate production (Rios-Covian et al., 2016). Although there is no data on fermentation yet, an interesting link between acidic phosphate groups in HePS structures and immune responses was found (Kitazawa et al., 1998). Lactobacillus delbrueckii subsp. bulgaricus OLL-1073-R1 produces two different EPS: acidic phosphate-containing (APS) and NPS, both composed of Glc and Gal residues (ratio 3:2). Interestingly, only the APS was a strong inducer of proliferation and activity of macrophages. When the APS was fractionated in two different EPS based on size, the B-cell mitogenic activity was observed only with high-molecular weight polysaccharide (H-APS). The impact of the acidic phosphate was substantiated by chemical dephosphorylation, which resulted in a reduction of the stimulatory effect (Kitazawa et al., 1998). Interestingly, when unrelated dextran ( $\alpha$-Glc HoPS from Leuconostoc mesenteroides, Table 1) was chemically phosphorylated, the proliferation of lymphocytes was directly proportional to the phosphate content (Sato et al., 2004). Unfortunately, there is no information available on the fermentability of these charged EPS, which could shed a light on their prebiotic potential. Overall, the structural complexity of especially the HePS yields large promise for prebiotic potential, which warrants extra dedication to unraveling the molecular structure of prebiotic HePS to gain more insight in the structure-function relation.

\section{DEVELOPMENT OF NOVEL NDCS}

With the increasing interest and appreciation of the impact of dietary glycans on healthy microbiome development and overall human health, there is a tremendous surge in methods to produce existing and novel glycans. Chemical synthesis has the potential to generate well-defined carbohydrate structures, but reliable methods are not generally available, and especially not on the scale that would allow for biological evaluation. Enzymatic synthesis is more amenable to larger scale carbohydrate production, but also has its challenges. GTs have successfully been used in the synthesis of HMO structures in vitro (Chen et al., 2015; Yu et al., 2017a), but their application is hampered by the use of expensive nucleotide-activated sugars, and multi-enzyme substrate recycling systems are needed to prevent metabolites from inhibiting enzyme activity (Qin et al., 2016). Using bacterial cells as production factories however, major advancements in HMO production have been made and have resulted in FDA approval and commercialization of the major HMO 2'-fucosyllactose. Different methods are now available in
Saccharomyces cerevisiae (Yu et al., 2018) and Escherichia coli (Chin et al., 2017), and other HMO structures are expected to be produced in this way in the near future (Sprenger et al., 2017). Alternative methods rely on the use of GHs, which are able to perform a transglycosylation reaction next to glycosidic bond hydrolysis (Danby and Withers, 2016; Manas et al., 2018). In this way, well-known prebiotic fibers such as GOS are industrially produced by making use of $\beta$-galactosidase enzymes (Torres et al., 2010), and also FOS can be synthesized in this way (Karboune et al., 2018). This approach can also be used to decorate existing glycans with other sugars, and the generation of galactosylated, fucosylated (Zeuner et al., 2018), and sialylated glycans as HMO mimics have recently been reviewed (Zeuner et al., 2014). A variety of glycan acceptors, ranging from monosaccharides and lactose to Tn antigens (e.g., $N$-acetylgalactosamine-threonine conjugates), GOS, and HMOs have been described. This strategy has the potential to rapidly yield novel dietary glycans that display complex sugar building blocks (e.g., Sia, Fuc) that were previously difficult to obtain.

A successful example of this strategy is the production and biological evaluation of sialylated GOS (Sia-GOS, Table 1). Using a transsialidase from Trypanosoma cruzi and bovine $\kappa$-casein-derived GMP as the source of Sia, commercial GOS was decorated with $\alpha-2,3$-Sia residues to create mono-Sia-GOS (Wilbrink et al., 2015). These novel glycans were subsequently tested in a rat model of NEC, an intestinal disorder mainly observed in preterm infants, for which sialylated HMOs were found to protect (Jantscher-Krenn et al., 2012; Yu et al., 2017b). Interestingly, Sia-GOS significantly reduced the pathology score of NEC, with pooled HMO still being superior in terms of protection, while regular GOS supplementation and formula-feeding both resulted in the worst pathology scores (Autran et al., 2016). In separate fermentation studies, with a Sia-GOS batch produced by a GT-catalyzed sialylation, it was revealed that $B$. infantis ATCC 15697 was able to digest Sia-GOS, whereas B. adolescentis ATCC 15703 could not, highlighting the species-specific ability to metabolize HMOs and HMO mimics (Wang et al., 2015).

Using a similar strategy, chitin and chitosan (deacetylated at the amine) oligosaccharides were decorated with $\beta$-Gal residues (Black et al., 2014). The transglycosylation was performed with $\beta$-galactosidase from Lactobacillus plantarum with lactose as the Gal source, and different chitin and chitosan acceptors were decorated with one to three residues in a $\beta$ 1,4 linkage (Table 1). Especially the $\beta$-Gal-chitosan and GOS oligosaccharides were found to prevent enterotoxigenic E. coli K88 from adhering to porcine erythrocytes, in contrast to alphalinked GOS and $\alpha$-Gal-chitosan (Yan et al., 2017; Yan and Ganzle, 2018). It will be interesting to perform digestion studies of these novel $\beta$-Gal-chitosan glycans by bacteria to investigate their prebiotic effect.

\section{CONCLUDING REMARKS}

It is clear that the creation of a healthy infant microbiome is a delicate interplay of a variety of commensal bacteria, which 
can be beneficially influenced by oligosaccharides. Because the composition of the infant's microbiome can have a profound effect on adult life, there is a great potential for the addition of carbohydrates that mimic HMO functions. Promising better candidates that may substitute or be added to currently applied NDCs are the HePS, which have the potential to specifically enhance certain species. Also, as structural mimics of HMOs, fucosylated and sialylated oligosaccharides are expected to be applied in the near future. In the end, more knowledge of the presence of the biosynthetic machinery necessary to utilize specific oligosaccharides will pave the way for the development of novel NDCs with prebiotic effects.

\section{REFERENCES}

Aachary, A. A., and Prapulla, S. G. (2011). Xylooligosaccharides (XOS) as an emerging prebiotic: microbial synthesis, utilization, structural characterization, bioactive properties, and applications. Compr. Rev. Food Sci. Food Saf. 10, 2-16. doi: 10.1111/j.1541-4337.2010.00135.x

Absmanner, B., Schmeiser, V., Kaempf, M., and Lehle, L. (2010). Biochemical characterization, membrane association and identification of amino acids essential for the function of Alg11 from Saccharomyces cerevisiae, an alpha 1,2-mannosyltransferase catalysing two sequential glycosylation steps in the formation of the lipid-linked core oligosaccharide. Biochem. J. 426, 205-217. doi: 10.1042/BJ20091121

Ackerman, D. L., Craft, K. M., and Townsend, S. D. (2017). Infant food applications of complex carbohydrates: structure, synthesis, and function. Carbohydr. Res. 437, 16-27. doi: 10.1016/j.carres.2016.11.007

Akkerman, R., Faas, M. M., and de Vos, P. (2018). Non-digestible carbohydrates in infant formula as substitution for human milk oligosaccharide functions: effects on microbiota and gut maturation. Crit. Rev. Food Sci. Nutr. doi: 10. 1080/10408398.2017.1414030 [Epub ahead of print].

Autran, C. A., Schoterman, M. H. C., Jantscher-Krenn, E., Kamerling, J. P., and Bode, L. (2016). Sialylated galacto-oligosaccharides and 2-fucosyllactose reduce necrotising enterocolitis in neonatal rats. Br. J. Nutr. 116, 294-299. doi: 10.1017/ S0007114516002038

Belkaid, Y., and Hand, T. W. (2014). Role of the microbiota in immunity and inflammation. Cell 157, 121-141. doi: 10.1016/j.cell.2014. 03.011

Benitez-Paez, A., Gomez Del Pulgar, E. M., Kjolbaek, L., Brahe, L. K., Astrup, A., and Larsen, L. (2016). Impact of dietary fiber and fat on gut microbiota re-modeling and metabolic health. Trends Food Sci. Technol. 57, 201-212. doi: 10.1038/srep10604

Black, B. A., Yan, Y., Galle, S., Hu, Y., Curtis, J. M., and Gaenzle, M. G. (2014). Characterization of novel galactosylated chitin-oligosaccharides and chitosan-oligosaccharides. Int. Dairy J. 39, 330-335. doi: 10.1016/j.idairyj.2014. 08.001

Bode, L. (2012). Human milk oligosaccharides: every baby needs a sugar mama. Glycobiology 22, 1147-1162. doi: 10.1093/glycob/cws074

Carmen Collado, M., Rautava, S., Aakko, J., Isolauri, E., and Salminen, S. (2016). Human gut colonisation may be initiated in utero by distinct microbial communities in the placenta and amniotic fluid. Sci. Rep. 6:23129. doi: 10.1038/ srep23129

Castro-Bravo, N., Wells, J. M., Margolles, A., and Ruas-Madiedo, P. (2018). Interactions of surface exopolysaccharides from Bifidobacterium and Lactobacillus within the intestinal environment. Front. Microbiol. 9:2426. doi: 10.3389/fmicb.2018.02426

Chen, C., Zhang, Y., Xue, M., Liu, X. W., Li, Y., Chen, X., et al. (2015). Sequential one-pot multienzyme (OPME) synthesis of lacto-N-neotetraose and its sialyl and fucosyl derivatives. Chem. Commun. 51, 7689-7692. doi: 10.1039/ c5cc01330e

\section{AUTHOR CONTRIBUTIONS}

SV, MF, and MW contributed to the organization and structure of the review. All authors contributed to the writing and critical evaluation of the final version.

\section{FUNDING}

Within the framework of the Carbohydrate Competence Center, this research has been financially supported by the Netherlands Organization for Scientific Research (NWO). MW acknowledges financial support from the European Union through the Rosalind Franklin Fellowship COFUND project 600211.

Chin, Y., Kim, J., Kim, J., Jung, S., and Seo, J. (2017). Improved production of 2 '-fucosyllactose in engineered Escherichia coli by expressing putative alpha1,2-fucosyltransferase, wcf from Bacteroides fragilis. J. Biotechnol. 257, 192-198. doi: 10.1016/j.jbiotec.2016.11.033

Crider, K. S., Yang, T. P., Berry, R. J., and Bailey, L. B. (2012). Folate and DNA methylation: a review of molecular mechanisms and the evidence for folate's role. Adv. Nutr. 3, 21-38. doi: 10.3945/an.111.000992

Dal Bello, F., Walter, J., Hertel, C., and Hammes, W. (2001). In vitro study of prebiotic properties of levan-type exopolysaccharides from lactobacilli and non-digestible carbohydrates using denaturing gradient gel electrophoresis. Syst. Appl. Microbiol. 24, 232-237. doi: 10.1078/0723-2020-00033

Danby, P. M., and Withers, S. G. (2016). Advances in enzymatic glycoside synthesis. ACS Chem. Biol. 11, 1784-1794. doi: 10.1021/acschembio.6b00340

de Jesus Raposo, M. F., Miranda de Morais, A. M., and de Morais, R. M. (2016). Emergent sources of prebiotics: seaweeds and microalgae. Mar. Drugs 14:E27. doi: $10.3390 / \mathrm{md} 14020027$

De Vuyst, L., and Leroy, F. (2011). Cross-feeding between bifidobacteria and butyrate-producing colon bacteria explains bifdobacterial competitiveness, butyrate production, and gas production. Int. J. Food Microbiol. 149, 73-80. doi: 10.1016/j.ijfoodmicro.2011.03.003

Di, R., Vakkalanka, M. S., Onumpai, C., Chau, H. K., White, A., Rastall, R. A., et al. (2017). Pectic oligosaccharide structure-function relationships: prebiotics, inhibitors of Escherichia coli $\mathrm{O} 157: \mathrm{H} 7$ adhesion and reduction of shiga toxin cytotoxicity in HT29 cells. Food Chem. 227, 245-254. doi: 10.1016/j.foodchem. 2017.01.100

Engfer, M., Stahl, B., Finke, B., Sawatzki, G., and Daniel, H. (2000). Human milk oligosaccharides are resistant to enzymatic hydrolysis in the upper gastrointestinal tract. Am. J. Clin. Nutr. 71, 1589-1596. doi: 10.1093/ajcn/71. 6.1589

Fanaro, S., Jelinek, T., Stahl, T., Boehm, T., Kock, R., and Vigi, V. (2005). Acidic oligosaccharides from pectin hydrolysate as new component for infant formulae: effect on intestinal flora, stool characteristics, and pH. J. Pediatr. Gastroenterol. Nutr. 41, 186-190. doi: 10.1097/01.mpg.0000172747.64103.d7

Furusawa, Y., Obata, Y., Fukuda, S., Endo, T. A., Nakato, G., Takahashi, D., et al. (2013). Commensal microbe-derived butyrate induces the differentiation of colonic regulatory T cells. Nature 504, 446-450. doi: 10.1038/nature12721

Garrido, D., Barile, D., and Mills, D. A. (2012). A molecular basis for bifidobacterial enrichment in the infant gastrointestinal tract. Adv. Nutr. 3, 415S-421S. doi: 10.3945/an.111.001586

Gibson, G. R., Hutkins, R., Sanders, M. E., Prescott, S. L., Reimer, R. A., Salminen, S. J., et al. (2017). The international scientific association for probiotics and prebiotics (ISAPP) consensus statement on the definition and scope of prebiotics. Nat. Rev. Gastroenterol. Hepatol. 14, 491-502. doi: 10.1038/nrgastro. 2017.75

Gomez, B., Gullon, B., Yanez, R., Schols, H., and Alonso, J. L. (2016). Prebiotic potential of pectins and pectic oligosaccharides derived from lemon peel wastes and sugar beet pulp: a comparative evaluation. J. Funct. Foods 20, 108-121. doi: 10.1016/j.jff.2015.10.029 
Gonzalez-Perez, G., Hicks, A. L., Tekieli, T. M., Radens, C. M., Williams, B. L., and Lamouse-Smith, E. S. N. (2016). Maternal antibiotic treatment impacts development of the neonatal intestinal microbiome and antiviral immunity. J. Immunol. 196, 3768-3779. doi: 10.4049/jimmunol.1502322

Gröber, U., Reichrath, J., Holick, M. F., and Kisters, K. (2014). Vitamin K: an old vitamin in a new perspective. DermatoEndocrinol. 6:e968490. doi: 10.4161/ 19381972.2014.968490

Haarman, M., and Knol, J. (2005). Quantitative real-time PCR assays to identify and quantify fecal Bifidobacterium species in infants receiving a prebiotic infant formula. Appl. Environ. Microbiol. 71, 2318-2324. doi: 10.1128/AEM.71.5.23182324.2005

Hidalgo-Cantabrana, C., Sanchez, B., Milani, C., Ventura, M., Margolles, A., and Ruas-Madiedo, P. (2014). Genomic overview and biological functions of exopolysaccharide biosynthesis in Bifidobacterium spp. Appl. Environ. Microbiol. 80, 9-18. doi: 10.1128/AEM.02977-13

Houghteling, P. D., and Walker, W. A. (2015). Why is initial bacterial colonization of the intestine important to infants' and children's health? J. Pediatr. Gastroenterol. Nutr. 60, 294-307. doi: 10.1097/MPG.0000000000000597

Jantscher-Krenn, E., Zherebtsov, M., Nissan, C., Goth, K., Guner, Y. S., Naidu, N., et al. (2012). The human milk oligosaccharide disialyllacto-N-tetraose prevents necrotising enterocolitis in neonatal rats. Gut 61, 1417-1425. doi: 10.1136/ gutjnl-2011-301404

Karboune, S., Appanah, N., Khodaei, N., and Tian, F. (2018). Enzymatic synthesis of fructooligosaccharides from sucrose by endo-inulinase-catalyzed transfructosylation reaction in biphasic systems. Process Biochem. 69, 82-91. doi: 10.1016/j.procbio.2018.03.010

Kitazawa, H., Harata, T., Uemura, J., Saito, T., Kaneko, T., and Itoh, T. (1998). Phosphate group requirement for mitogenic activation of lymphocytes by an extracellular phosphopolysaccharide from Lactobacillus delbrueckii ssp. bulgaricus. Int. J. Food Microbiol. 40, 169-175. doi: 10.1016/S0168-1605(98) 00030-0

Korakli, M., Ganzle, M., and Vogel, R. (2002). Metabolism by bifidobacteria and lactic acid bacteria of polysaccharides from wheat and rye, and exopolysaccharides produced by Lactobacillus sanfranciscensis. J. Appl. Microbiol. 92, 958-965. doi: 10.1046/j.1365-2672.2002.01607.x

Lecerf, J., Depeint, F., Clerc, E., Dugenet, Y., Niamba, C. N., Rhazi, L., et al. (2012). Xylo-oligosaccharide (XOS) in combination with inulin modulates both the intestinal environment and immune status in healthy subjects, while XOS alone only shows prebiotic properties. Br. J. Nutr. 108, 1847-1858. doi: 10.1017/ S0007114511007252

Leijdekkers, A. G. M., Aguirre, M., Venema, K., Bosch, G., Gruppen, H., and Schols, H. A. (2014). In vitro fermentability of sugar beet pulp derived oligosaccharides using human and pig fecal inocula. J. Agric. Food Chem. 62, 1079-1087. doi: $10.1021 /$ jf 4049676

LoCascio, R. G., Ninonuevo, M. R., Kronewitter, S. R., Freeman, S. L., German, J. B., Lebrilla, C. B., et al. (2009). A versatile and scalable strategy for glycoprofiling bifidobacterial consumption of human milk oligosaccharides. Microb. Biotechnol. 2, 333-342. doi: 10.1111/j.1751-7915.2008.00072.x

Lozupone, C. A., Stombaugh, J. I., Gordon, J. I., Jansson, J. K., and Knight, R. (2012). Diversity, stability and resilience of the human gut microbiota. Nature 489, 220-230. doi: 10.1038/nature 11550

Macfarlane, G. T., Steed, H., and Macfarlane, S. (2008). Bacterial metabolism and health-related effects of galacto-oligosaccharides and other prebiotics. J. Appl. Microbiol. 104, 305-344. doi: 10.1111/j.1365-2672.2007.03520.x

Manas, N. H. A., Illias, R. M., and Mahadi, N. M. (2018). Strategy in manipulating transglycosylation activity of glycosyl hydrolase for oligosaccharide production. Crit. Rev. Biotechnol. 38, 272-293. doi: 10.1080/07388551.2017.1339664

Marcobal, A., Barboza, M., Froehlich, J. W., Block, D. E., German, J. B., Lebrilla, C. B., et al. (2010). Consumption of human milk oligosaccharides by gut-related microbes. J. Agric. Food Chem. 58, 5334-5340. doi: 10.1021/ jf9044205

Martin, R., Nauta, A. J., Ben Amor, K., Knippels, L. M. J., Knol, J., and Garssen, J. (2010). Early life: gut microbiota and immune development in infancy. Benef. Microbes 1, 367-382. doi: 10.3920/BM2010.0027

Milani, C., Duranti, S., Bottacini, F., Casey, E., Turroni, F., Mahony, J., et al. (2017). The first microbial colonizers of the human gut: composition, activities, and health implications of the infant gut microbiota. Microbiol. Mol. Biol. Rev. 81:e00036-17. doi: 10.1128/MMBR.00036-17
Moens, F., Weckx, S., and De Vuyst, L. (2016). Bifidobacterial inulin-type fructan degradation capacity determines cross-feeding interactions between bifidobacteria and Faecalibacterium prausnitzii. Int. J. Food Microbiol. 231, 76-85. doi: 10.1016/j.ijfoodmicro.2016.05.015

Nijman, R. M., Liu, Y., Bunyatratchata, A., Smilowitz, J. T., Stahl, B., and Barile, D. (2018). Characterization and quantification of oligosaccharides in human milk and infant formula. J. Agric. Food Chem. 66, 6851-6859. doi: 10.1021/acs.jafc. 8 b01515

Oleksy, M., and Klewicka, E. (2018). Exopolysaccharides produced by Lactobacillus sp.: biosynthesis and applications. Crit. Rev. Food Sci. Nutr. 58, 450-462. doi: 10.1080/10408398.2016.1187112

Onumpai, C., Kolida, S., Bonnin, E., and Rastall, R. A. (2011). Microbial utilization and selectivity of pectin fractions with various structures. Appl. Environ. Microbiol. 77, 5747-5754. doi: 10.1128/AEM.00179-11

Paparo, L., di Costanzo, M., di Scala, C., Cosenza, L., Leone, L., Nocerino, R., et al. (2014). The influence of early life nutrition on epigenetic regulatory mechanisms of the immune system. Nutrients 6, 4706-4719. doi: 10.3390/ nu6114706

Qin, H., Li, S., Zhang, Y., Wang, J. W., Li, J., Song, S., et al. (2016). Multienzymatic cascade synthesis of fucosyloligosaccharide via a two-step fermentation strategy in Escherichia coli. Biotechnol. Lett. 38, 1747-1752. doi: 10.1007/s10529-0162151-y

Ramiro do Carmo, M. M., Leite Walker, J. C., Novello, D., Caselato, V. M., Sgarbieri, V. C., Ouwehand, A. C., et al. (2016). Polydextrose: physiological function, and effects on health. Nutrients 8:E553. doi: 10.3390/nu8090553

Rios-Covian, D., Cuesta, I., Alvarez-Buylla, J. R., Ruas-Madiedo, P., Gueimonde, M., and de los Reyes-Gavilan, C. G. (2016). Bacteroides fragilis metabolises exopolysaccharides produced by bifidobacteria. BMC Microbiol. 16:150. doi: 10.1186/s12866-016-0773-9

Rivas, S., Santos, V., and Parajo, J. C. (2017). Aqueous fractionation of hardwood: selective glucuronoxylan solubilisation and purification of the reaction products. J. Chem. Technol. Biotechnol. 92, 367-374. doi: 10.1002/jctb. 5014

Riviere, A., Gagnon, M., Weckx, S., Roy, D., and De Vuyst, L. (2015). Mutual crossfeeding interactions between Bifidobacterium longum subsp longum NCC2705 and eubacterium rectale ATCC 33656 explain the bifidogenic and butyrogenic effects of arabinoxylan oligosaccharides. Appl. Environ. Microbiol. 81, 77677781. doi: 10.1128/AEM.02089-15

Riviere, A., Selak, M., Geirnaert, A., Van den Abbeele, P., and De Vuyst, L. (2018). Complementary mechanisms for degradation of inulin-type fructans and arabinoxylan oligosaccharides among bifidobacterial strains suggest bacterial cooperation. Appl. Environ. Microbiol. 84:e02893-17. doi: 10.1128/AEM. 02893-17

Riviere, A., Selak, M., Lantin, D., Leroy, F., and De Vuyst, L. (2016). Bifidobacteria and butyrate-producing colon bacteria: importance and strategies for their stimulation in the human gut. Front. Microbiol. 7:979. doi: 10.3389/fmicb.2016. 00979

Rossi, M., Amaretti, A., and Raimondi, S. (2011). Folate production by probiotic bacteria. Nutrients 3, 118-134. doi: 10.3390/nu3010118

Ryan, P. M., Ross, R. P., Fitzgerald, G. F., Caplice, N. M., and Stanton, C. (2015). Sugar-coated: exopolysaccharide producing lactic acid bacteria for food and human health applications. Food Funct. 6, 679-693. doi: 10.1039/c4fo00529e

Salazar, N., Gueimonde, M., de los Reyes-Gavilan, C. G., and Ruas-Madiedo, P. (2016). Exopolysaccharides produced by lactic acid bacteria and bifidobacteria as fermentable substrates by the intestinal microbiota. Crit. Rev. Food Sci. Nutr. 56, 1440-1453. doi: 10.1080/10408398.2013.770728

Salazar, N., Gueimonde, M., Maria Hernandez-Barranco, A., Ruas-Madiedo, P., and Reyes-Gavilan, C. G. (2008). Exopolysaccharides produced by intestinal Bifidobacterium strains act as fermentable substrates for human intestinal bacteria. Appl. Environ. Microbiol. 74, 4737-4745. doi: 10.1128/AEM.00325-08

Sato, T., Nishimura-Uemura, J., Shimosato, T., Kawai, Y., Kitazawa, H., and Saito, T. (2004). Dextran from leuconostoc mesenteroides augments immunostimulatory effects by the introduction of phosphate groups. J. Food Prot. 67, 1719-1724. doi: 10.4315/0362-028X-67.8.1719

Scalabrin, D. M. F., Mitmesser, S. H., Welling, G. W., Harris, C. L., Marunycz, J. D., Walker, D. C., et al. (2012). New prebiotic blend of polydextrose and galacto-oligosaccharides has a bifidogenic effect in young infants. J. Pediatr. Gastroenterol. Nutr. 54, 343-352. doi: 10.1097/MPG.0b013e318237ed95 
Sela, D. A., and Mills, D. A. (2010). Nursing our microbiota: molecular linkages between bifidobacteria and milk oligosaccharides. Trends Microbiol. 18 298-307. doi: 10.1016/j.tim.2010.03.008

Sender, R., Fuchs, S., and Milo, R. (2016). Revised estimates for the number of human and bacteria cells in the body. PLoS Biol. 14:e1002533. doi: 10.1371/ journal.pbio. 1002533

Sierra, C., Bernal, M., Blasco, J., Martínez, R., Dalmau, J., Ortuño, I., et al. (2015). Prebiotic effect during the first year of life in healthy infants fed formula containing GOS as the only prebiotic: a multicentre, randomised, double-blind and placebo-controlled trial. Eur. J. Nutr. 54, 89-99. doi: 10.1007/s00394-0140689-9

Sprenger, G. A., Baumgaertner, F., and Albermann, C. (2017). Production of human milk oligosaccharides by enzymatic and whole-cell microbial biotransformations. J. Biotechnol. 258, 79-91. doi: 10.1016/j.jbiotec.2017.07.030

Stilling, R. M., van de Wouw, M., Clarke, G., Stanton, C., Dinan, T. G., and Cryan, J. F. (2016). The neuropharmacology of butyrate: the bread and butter of the microbiota-gut-brain axis? Neurochem. Int. 99, 110-132. doi: 10.1016/j.neuint. 2016.06.011

Thakur, K., Tomar, S. K., and De, S. (2016). Lactic acid bacteria as a cell factory for riboflavin production. Microb. Biotechnol. 9, 441-451. doi: 10.1111/1751-7915. 12335

Thomson, P., Medina, D. A., and Garrido, D. (2018). Human milk oligosaccharides and infant gut bifidobacteria: molecular strategies for their utilization. Food Microbiol. 75, 37-46. doi: 10.1016/j.fm.2017.09.001

Torres, D. P. M., Goncalves, M. P., Teixeira, J. A., and Rodrigues, L. R. (2010). Galacto-oligosaccharides: production, properties, applications, and significance as prebiotics. Compr. Rev. Food Sci. Food Saf. 9, 438-454. doi: 10.1111/j.15414337.2010.00119.x

Vandenplas, Y., Zakharova, I., and Dmitrieva, Y. (2015). Oligosaccharides in infant formula: more evidence to validate the role of prebiotics. Br. J. Nutr. 113, 1339-1344. doi: 10.1017/S0007114515000823

Vernazza, C., Gibson, G., and Rastall, R. (2006). Carbohydrate preference, acid tolerance and bile tolerance in five strains of bifidobacterium. J. Appl. Microbiol. 100, 846-853. doi: 10.1111/j.1365-2672.2006.02832.x

Wang, B., Yao, M., Lv, L., Ling, Z., and Li, L. (2017). The human microbiota in health and disease. Engineering 3, 71-82. doi: 10.1016/J.ENG.2017.01.008

Wang, Y., Jiang, K., Ma, H., Zeng, W., Wang, P. G., Yao, N., et al. (2015). Enzymatic production of HMO mimics by the sialylation of galacto-oligosaccharides. Food Chem. 181, 51-56. doi: 10.1016/j.foodchem.2015.02.064

Wilbrink, M. H., ten Kate, G. A., Sanders, P., Gerwig, G. J., van Leeuwen, S. S., Sallomons, E., et al. (2015). Enzymatic decoration of prebiotic galacto-oligosaccharides (vivinal GOS) with sialic acid using trypanosoma cruzi trans-sialidase and two bovine sialoglycoconjugates as donor substrates. J. Agric. Food Chem. 63, 5976-5984. doi: 10.1021/acs.jafc.5b01505

Wu, X., Wu, Y., He, L., Wu, L., Wang, X., and Liu, Z. (2018). Effects of the intestinal microbial metabolite butyrate on the development of colorectal cancer. J. Cancer 9, 2510-2517. doi: 10.7150/jca.25324

Yan, Y. L., and Ganzle, M. G. (2018). Structure and function relationships of the binding of beta- and alpha-galactosylated oligosaccharides to K88 fimbriae of enterotoxigenic Escherichia coli. Int. Dairy J. 81, 104-112. doi: 10.1016/j.idairyj. 2018.01.006

Yan, Y. L., Hu, Y., Simpson, D. J., and Ganzle, M. G. (2017). Enzymatic synthesis and purification of galactosylated chitosan oligosaccharides reducing adhesion of enterotoxigenic Escherichia coli K88. J. Agric. Food Chem. 65, 5142-5150. doi: 10.1021/acs.jafc.7b01741

Yang, I., Corwin, E. J., Brennan, P. A., Jordan, S., Murphy, J. R., and Dunlop, A. (2016). The infant microbiome implications for infant health and neurocognitive development. Nurs. Res. 65, 76-88. doi: 10.1097/NNR. 0000000000000133

Yu, H., Li, Y., Wu, Z., Li, L., Zeng, J., Zhao, C., et al. (2017a). H-pylori alpha 1-3/4-fucosyltransferase (Hp3/4FT)-catalyzed one-pot multienzyme (OPME) synthesis of lewis antigens and human milk fucosides. Chem. Commun. 53, 11012-11015. doi: 10.1039/c7cc05403c

Yu, H., Yan, X., Autran, C. A., Li, Y., Etzold, S., Latasiewicz, J., et al. (2017b). Enzymatic and chemoenzymatic syntheses of disialyl glycans and their necrotizing enterocolitis preventing effects. J. Org. Chem. 82, 13152-13160. doi: 10.1021/acs.joc.7b02167

Yu, S., Liu, J., Yun, E. J., Kwak, S., Kim, K. H., and Jin, Y. (2018). Production of a human milk oligosaccharide 2 '-fucosyllactose by metabolically engineered Saccharomyces cerevisiae. Microb. Cell Fact. 17:101. doi: 10.1186/s12934-0180947-2

Zeuner, B., Jers, C., Mikkelsen, J. D., and Meyer, A. S. (2014). Methods for improving enzymatic trans-glycosylation for synthesis of human milk oligosaccharide biomimetics. J. Agric. Food Chem. 62, 9615-9631. doi: 10.1021/ jf502619p

Zeuner, B., Muschiol, J., Holck, J., Lezyk, M., Gedde, M. R., Jers, C., et al. (2018). Substrate specificity and transfucosylation activity of GH29 alpha-L-fucosidases for enzymatic production of human milk oligosaccharides. New Biotechnol. 41, 34-45. doi: 10.1016/j.nbt.2017.12.002

Zhang, T., Yang, Y., Liang, Y., Jiao, X., and Zhao, C. (2018). Beneficial effect of intestinal fermentation of natural polysaccharides. Nutrients 10:E1055 doi: 10.3390/nu10081055

Zhao, J., and Cheung, P. C. K. (2011). Fermentation of beta-glucans derived from different sources by bifidobacteria: evaluation of their bifidogenic effect. J. Agric. Food Chem. 59, 5986-5992. doi: 10.1021/ jf200621y

Conflict of Interest Statement: The authors declare that the research was conducted in the absence of any commercial or financial relationships that could be construed as a potential conflict of interest.

Copyright (c) 2019 Verkhnyatskaya, Ferrari, de Vos and Walvoort. This is an openaccess article distributed under the terms of the Creative Commons Attribution License (CC BY). The use, distribution or reproduction in other forums is permitted, provided the original author(s) and the copyright owner(s) are credited and that the original publication in this journal is cited, in accordance with accepted academic practice. No use, distribution or reproduction is permitted which does not comply with these terms. 